\title{
CONODONTOS (BIOESTRATIGRAFÍA, BIOFACIES Y PALEOTEMPERATURAS) DE LOS SINCLINALES DE ALMADÉN Y GUADALMEZ (DEVÓNICO-CARBONÍFERO INFERIOR), ZONA CENTROIBÉRICA MERIDIONAL, ESPAÑA
}

\author{
Susana GARCÍA-LÓPEZ', Javier SANZ LÓPEZ² \\ y Miguel V. PARDO ALONSO \\ '. Facultad de Geología, Universidad de Oviedo. Arias de Velasco s/n. 33005 Oviedo. \\ 2. Facultade de Ciencias da Educación, Universidade da Coruña. Paseo de Ronda 47. 15011 A Coruña. \\ 3 Departamento de Geología, Universitat de Valencia. Dr. Moliner 50. E-46100 Burjassot. Valencia.
}

García-López, S., Sanz López, J. y Pardo Alonso, M.V. 1999. Conodontos (bioestratigrafía, biofacies y paleotemperaturas) de los sinclinales de Almadén y Guadalmez (Devónico-Carbonífero Inferior), Zona Centroibérica meridional, España. [Conodonts (biostratigraphy, biofacies and paleotemperatures) from Almadén and Guadalmez synclines (Devonian-Lower Carboniferous), southern Central Iberian Zone, Spain]. Revista Española de Paleontología, $\mathbf{n}^{\circ}$ extr. Homenaje al Prof. J. Truyols, 161-172. ISSN 0213-6937.

\begin{abstract}
Published and unpublished data on Devonian and Lower Carboniferous conodonts from the Almadén and Guadalmez synclines (southern Central-Iberian Zone) have been collated and revised, and their biostratigraphic, palaeoecological and palaeothermal applications analysed. Pre-Famennian Devonian conodont faunas are poor and exhibit a low diversity, indicating shallow water conditions with moderate to high energy. The fossil assemblages can be dated as late Emsian, late Givetian with some reservation, and late Frasnian. In the Famennian, the conodont faunas are abundant and show moderate diversity. Study of their biofacies indicates a increase in both water depth and distance from the coast. Conodont biozones are established for the first time for the early Famennian, i.e. the age attributed to the basal part of the Casa de la Vega Formation. Faunal gaps indicate two intervals of extreme condensation or an absence of stratigraphic record for middle Famennian, most of the upper Famennian, and at the Devonian-Carnoniferous boundary. The conodont colour alteration index (CAI) indicates an increasing metamorphic grade towards the south in the Almadén syncline. Transition from diagenesis to metamorphism occurs in Devonian rocks between the northern limb and core of the syncline. Diagenetic conditions have been recognised for the Guadalmez syncline, with a weak cleavage development in the southern limb which is associated with CAI values between 3 and 3.5. Conodont mineral phase (apatite) recrystallises for CAI values of 4.5 .
\end{abstract}

Keywords: Conodonts, biostratigraphy, biofacies, CAI, Devonian, Lower Carboniferous, southern Central-Iberian Zone, Iberian Massif, Spain.

\section{RESUMEN}

Se han reunido y revisado los datos (publicados e inéditos) sobre los conodontos del Devónico y Carbonífero Inferior de los sinclinales de Almadén y Guadalmez (Zona Centroibérica meridional, España). Se recogen las aplicaciones que tienen desde el punto de vista bioestratigráfico, paleoecológico y paleotérmico. En el Devónico pre-Fameniense, las faunas de conodontos son pobres y de baja diversidad e indican medios someros de alta a moderada energía; las asociaciones fósiles permiten identificar el Emsiense superior, el Givetiense superior con algunas reservas y el Frasniense superior. En el Fameniense, las faunas de conodontos son abundantes y de moderada diversidad y el estudio de sus biofacies indica un aumento de la profundidad y distancia a la costa en el medio deposicional. Se establecen por primera vez biozonas de conodontos en el Fameniense inferior, edad asignada a la base de la Fm. Casa de la Vega. Discontinuidades faunísticas durante el Fameniense medio y casi todo el Fameniense superior, así como en torno al límite Devónico-Carbonífero reflejan dos intervalos de extrema condensación o falta de registro estratigráfico. El índice de alteración del color de los conodontos (CAI) indica un metamorfismo creciente hacia el sur en el sinclinal de Almadén, localizándose la transición diagénesisanquizona en las rocas devónicas, entre el flanco norte y núcleo de esta estructura; para el sinclinal de 
Guadalmez se establecen condiciones diagenéticas, con el desarrollo de un débil clivaje en el flanco sur asociado a valores de CAI comprendidos entre 3 y 3, 5. Asimismo la fase mineral de los conodontos (apatito) recristaliza con valores de $\mathrm{CAI}=4,5$.

\section{Palabras clave: Conodontos, bioestratigrafía, biofacies, CAI, Devónico, Carbonífero Inferior, Zona Centroibérica meridional, Macizo Ibérico, España.}

\section{ANTECEDENTES}

Las sucesiones devónicas más completas descritas en la Zona Centroibérica (ZCI) meridional se localizan en los sinclinales de Almadén y Guadalmez, aflorando también materiales del Carbonífero Inferior en el núcleo de esta última estructura. Los conodontos estudiados en el presente trabajo proceden de diferentes niveles situados en el flanco norte y en el núcleo de ambos sinclinales (Fig. 1). Las unidades litoestratigráficas que hemos adoptado se encuentran esquematizadas en Pardo Alonso y García-Alcalde (1996) y un análisis más detallado en Pardo Alonso ( 1997), donde se propone una definición formal de las mismas.

El contenido fosilífero de los niveles devónicos es conocido desde antiguo (Verneuil y Barrande, 1855), aunque no ha sido estudiado con cierto detalle hasta época reciente (Pardo Alonso y García-Alcalde, 1984) y sólo en lo referente a los macrofósiles, principalmente braquiópodos. La primera indicación de la existencia de conodontos en el Devónico de la ZCI se debe a Puschmann (1970) quien, con determinaciones de P. Carls, citó nueve especies del Praguiense al Emsiense, procedentes del sinclinal de Herrera del Duque (al $\mathrm{N}$ de nuestra zona); el material fue extraído de niveles calcáreos situados en el entorno de la laguna estratigráfica del Devónico Medio, característica de la serie devónica en la ZCI meridional. En el mismo sinclinal, Calvo (1993) citó y describió varias especies de conodontos del Praguiense, procedentes de las "Calizas de Herrera" (Mb. Molino de la
Dehesa, en sentido actual).

En el área meridional, los conodontos no fueron citados hasta el trabajo de Vergés (1983) sobre el sinclinal de Almadén, mientras que listados más extensos con material de los sinclinales de Almadén y Guadalmez se encuentran en García-Alcalde et al. (1984) y Pardo Alonso y García-Alcalde (1984). En todos estos trabajos, las asociaciones obtenidas, en su mayor parte determinadas por uno de nosotros (S.G.-L.), permitieron precisar la edad de los niveles más altos del Devónico del sinclinal de Almadén, y también caracterizar el Fameniense y el límite Devónico-Carbonífero en el de Guadalmez.

El presente trabajo es una revisión de estas últimas determinaciones y su estudio más completo, con el añadido de nuevo material procedente de muestreos recientes, realizados durante la elaboración de la Hoja del MAGNA correspondiente a Chillón y los trabajos de campo de la tesis doctoral de uno de nosotros (M.V.P.A.). Se procede por primera vez a la asignación bioestratigráfica de los taxones de conodontos encontrados, lo que permite el establecimiento de una cronoestratigrafía más precisa, especialmente durante el Fameniense. Asimismo, el reconocimiento de biofacies nos da algunas indicaciones con relación a los medios de depósito, y la determinación del índice de alteración del color de los conodontos (CAI) permite discutir su relación con el grado de metamorfismo presente en la zona. Los resultados preliminares de esta investigación se encuentran en García-López et al. (1997, 1998).

\section{Lámina I}

1-2 Palmatolepis wolskajae Ovnatanova, 1969. x 55, muestra NG-5, Fm. Casa de la Vega, sinclinal de Guadalmez.

3 Palmatolepis triangularis $\rightarrow$ P. wolskajae. x 80, muestra NG-5, Fm. Casa de la Vega, sinclinal de Guadalmez.

4 Palmatolepis tenuipunctata Sannemann, 1955. x 50, muestra F-218, Fm. Casa de la Vega, sinclinal de Guadalmez.

5 Palmatolepis quadrantinodosalobata Sannemann, 1955. x 50, muestra G-13, Fm. Casa de la Vega, sinclinal de Guadalmez.

6 Palmatolepis termini Sannemann 1955. x 85, muestra G-13, Fm. Casa de la Vega, Sinclinal de Guadalmez.

7 Branmelha inornata (Branson y Mehl, 1934). x 50, muestra PG-6, Fm. Casa de la Vega, corte del Pantano de Mendoza, sinclinal de Guadalmez.

8 Palmatolepis rhomboidea Sannemann, 1955. x 80, muestra PG-5, Fm. Casa de la Vega, corte del Pantano de Mendoza, sinclinal de Guadalmez.

9 Palmatolepis minuta minuta Branson y Mehl, 1934.x

110, muestra PG-5, Fm. Casa de la Vega, corte del Pantano de Mendoza, sinclinal de Guadalmez.

10 Palmatolepis gracilis gracilis Branson y Mehl, 1934. x 60, muestra PG-6, Fm. Casa de la Vega, corte del Pantano de Mendoza, sinclinal de Guadalmez.

11 Ozarkodina steinhornensis steinhornensis (Ziegler, 1956). x 90, muestra MP-24, Fm. Herrera, Mb. Molino de la Dehesa, sinclinal de Almadén.

12-14 Mehlina sp. 12-13, elementos Pa x 110; 14, elemento $\mathrm{Pb}$ x 140. Presentan patología tipo "descompositio", muestra NG-5, Fm. Casa de la Vega, sinclinal de Guadalmez.

15 Ancyrognathus sinelaminus (Branson y Mehl, 1934). x 35, muestra NG-5, Fm. Casa de la Vega, sinclinal de Guadalmez.

16 Gnathodus semiglaber Bischoff, 1957. x 35, muestra PG-7, Fm. Casa de la Vega, corte del Pantano de Mendoza, sinclinal de Guadalmez.

17-18 Gnathodus pseudosemiglaber Thompson y Fellows, 1970. x 70, muestra G-3, Fm. Casa de la Vega, corte del Pantano de Mendoza, sinclinal de Guadalmez. 
Lámina I

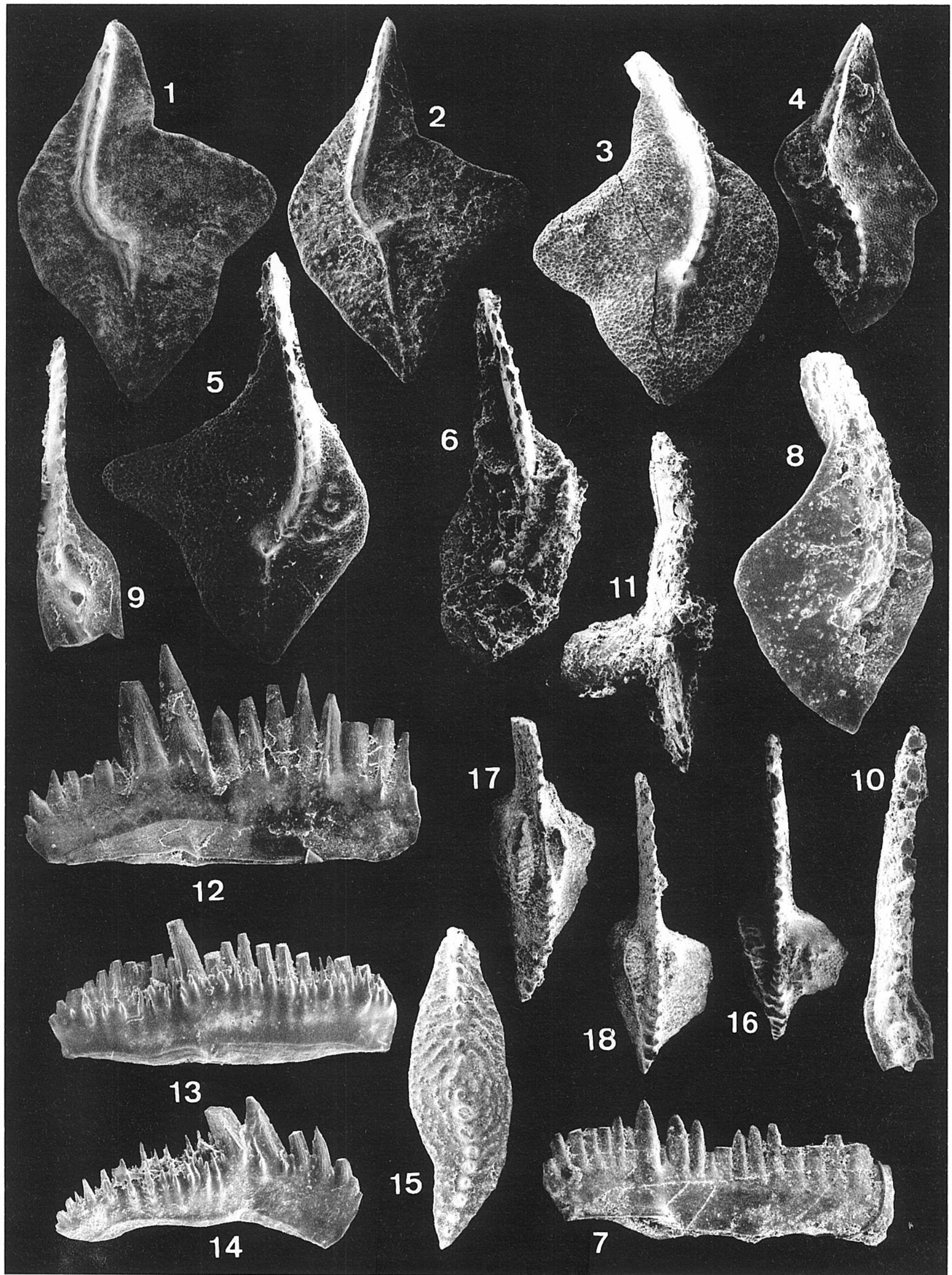

Revista Española de Paleontología, no extr. Homenaje al Prof. J. Truyols, 1999. 


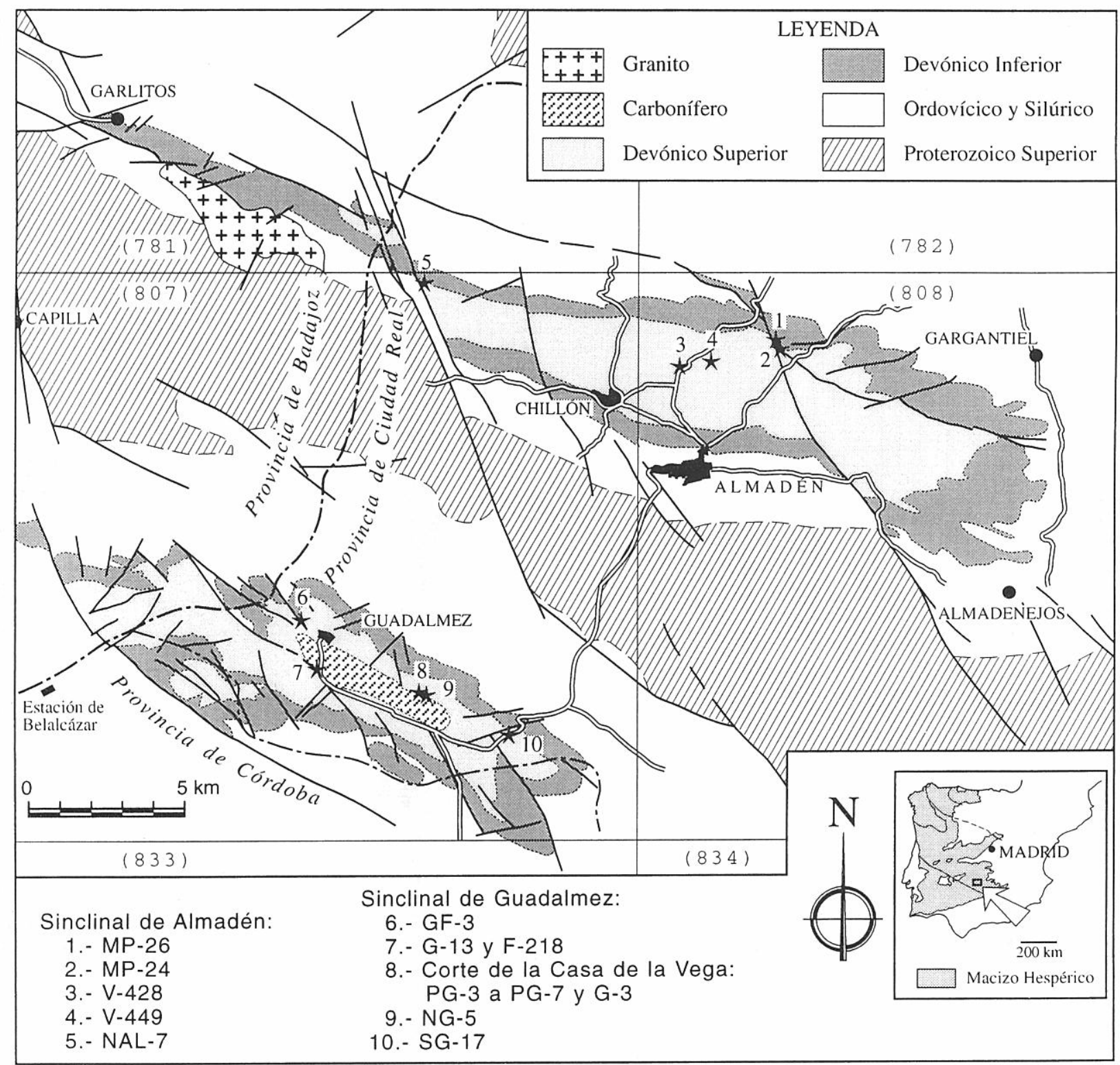

Figura 1. Mapa geológico de los sinclinales de Almadén y Guadalmez y situación de las localidades con conodontos. Abajo a la derecha, posición de la zona dentro del Macizo Ibérico. Base cartográfica, Pardo Alonso (1997).

\section{FAUNAS DE CONODONTOS}

Los conodontos devónicos de la región han sido poco estudiados, contándose apenas con listados de las especies determinadas; esta omisión se debe no sólo al poco desarrollo que han tenido los estudios paleontológicos en el Devónico regional hasta época reciente, sino también a la escasez de niveles carbonatados de donde poder extraerlos por métodos tradicionales.

En la series estudiadas, los niveles de calizas (sensu stricto) se localizan en dos unidades muy concretas: el Mb. Molino de la Dehesa (Praguiense/Emsiense) de la Fm. Herrera, y la Fm. Casa de la Vega (Fameniense /Viseense). Otras tres unidades frasnienses, las fms. Abulagar, Valmayor y el Complejo vulcano-sedimentario de Chillón, aunque constituídas mayoritariamente por sedimentos detríticos y vulcano-sedimentarios, contienen localmente niveles lenticulares de areniscas carbonatadas bioclásticas, y calizas bioclásticas muy arenosas, que también han sido muestreadas para la extracción de conodontos. En todas las unidades citadas se han encontrado conodontos, aunque no todas las muestras procesadas han dado resultados positivos. Los conodontos y microfauna asociada están depositados en el Departamento de Geología de la Universidad de Oviedo.

\section{SINCLINAL DE ALMADÉN}

Los muestreos se han realizado en el flanco norte y núcleo de esta estructura (Fig. 1). La cantidad de roca tratada para cada muestra fue de unos 500 gr y los conodontos son escasos, entre 2 y 6 elementos por muestra. Se han encontrado conodontos en el Miembro Molino de la Dehesa de la Fm. Herrera, en los tramos basales de la Fm. Abulagar y dentro del Complejo vulcano-sedimentario de Chillón (Fig. 2). 


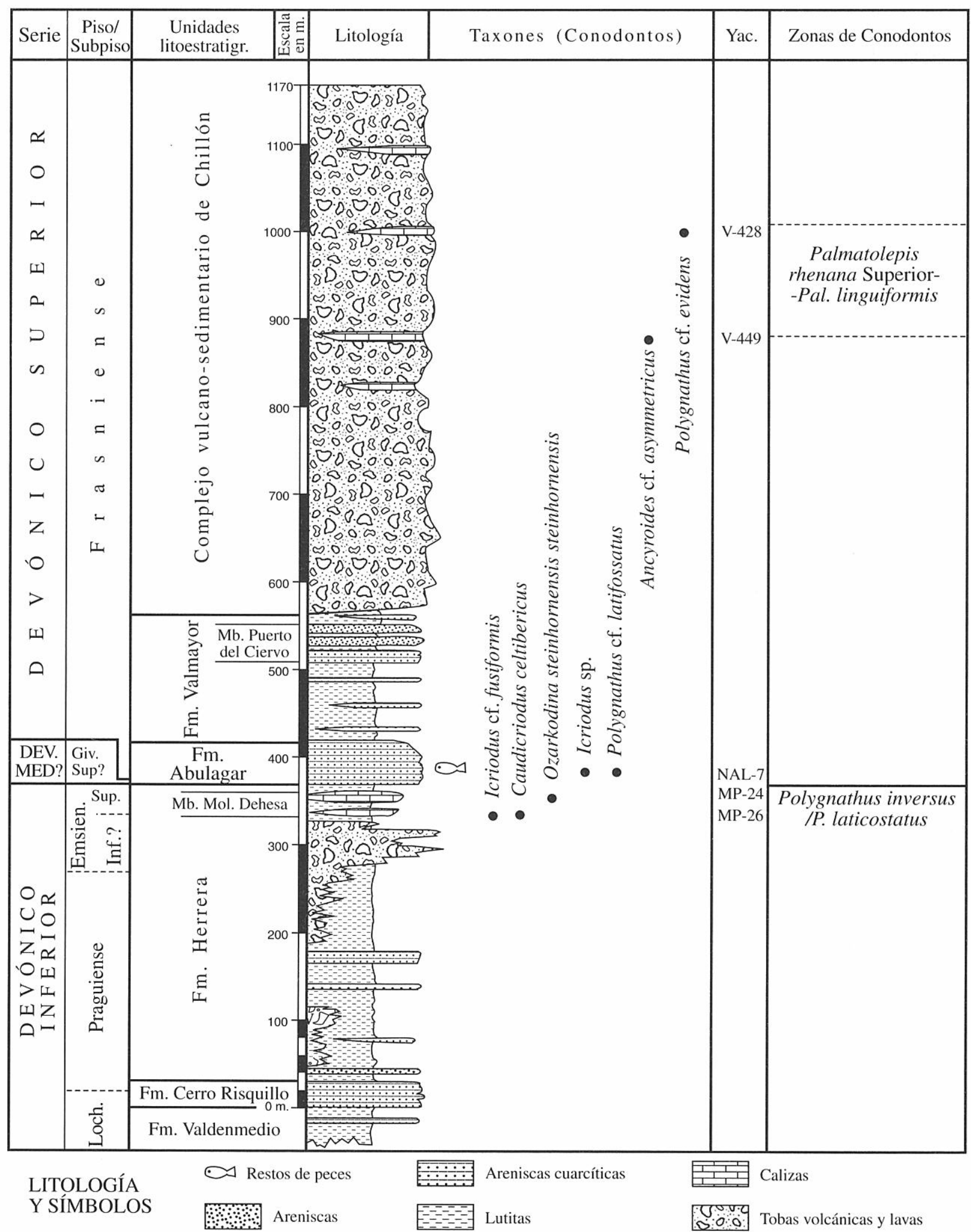

Figura 2. Columna sintética del Devónico del flanco norte del sinclinal de Almadén, con indicación de la posición estratigráfica de las especies de conodontos referidas en el texto.

El Miembro Molino de la Dehesa de la Fm. Herrera aflora únicamente en el sinclinal de Almadén y está constituído por calizas bioclásticas, margosas unas veces, arenosas otras, con abundantes braquiópodos, y en menor medida briozoos, crinoideos y otra macrofauna bentónica.
Cerca del Arroyo del Enjambradero, la capa basal MP-26 ha suministrado Icriodus cf. fusiformis Carls y Gandl y Caudicriodus celtibericus (Carls y Gandl); una capa suprayacente, MP-24 contiene Ozarkodina steinhornensis steinhornensis (Ziegler) (Lám. I, fig 11). Esta fauna 


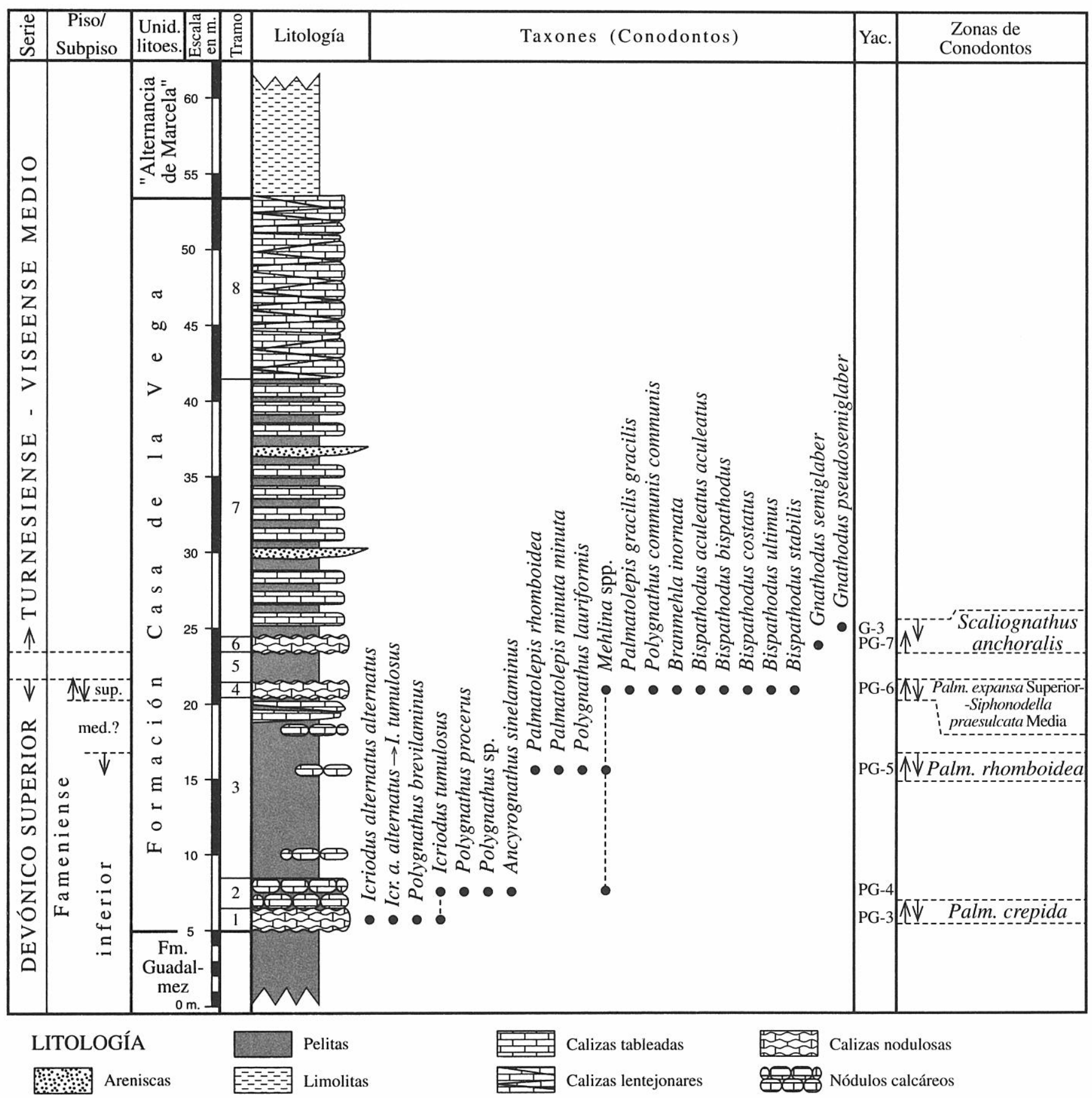

Figura 3. Columna del Pantano de Mendoza (Guadalmez). Niveles carbonatados del Devónico Superior (Fameniense) y del Carbonífero Inferior y contenido en conodontos de los mismos.

podría corresponder a la Zona de Polygnathus inversus/P. laticostatus de Bultynck (1989) del Emsiense superior (Fig. 2).

La Formación Abulagar en sus tramos basales está constituída por calizas arenosas bioclásticas, de tonos oscuros, con fragmentos de limolitas violáceas y grises. En la muestra NAL-7 se han encontrado únicamente pequeños ejemplares de Icriodus sp. (Lám. II, figs. 15-16), junto a un ejemplar de Polygnathus, con una cavidad basal expandida, identificado provisionalmente como Polygnathus cf. latifossatus Wirth. Este hallazgo es atribuído al Givetiense superior, sin mucha precisión (Fig. 2).
El Complejo vulcano-sedimentario de Chillón está integrado por rocas casi exclusivamente volcánicas, con algunas rocas siliciclásticas y carbonatadas de extensión lateral escasa (Fig. 2). Dos niveles calcáreos, V-449 y V428, han proporcionado Ancyroides cf. asymmetricus (Ulrich y Bassler) y Polygnathus cf. evidens Klapper y Lane (Lám. II, fig. 1). El intervalo comprendido entre ambos niveles puede corresponder a un horizonte u horizontes entre la Zona de Palmatolepis rhenana Superior de Ziegler y Sandberg (1990) y la parte más alta de la Zona de Palmatolepis linguiformis de Sandberg el al. (1988) del Frasniense superior. 


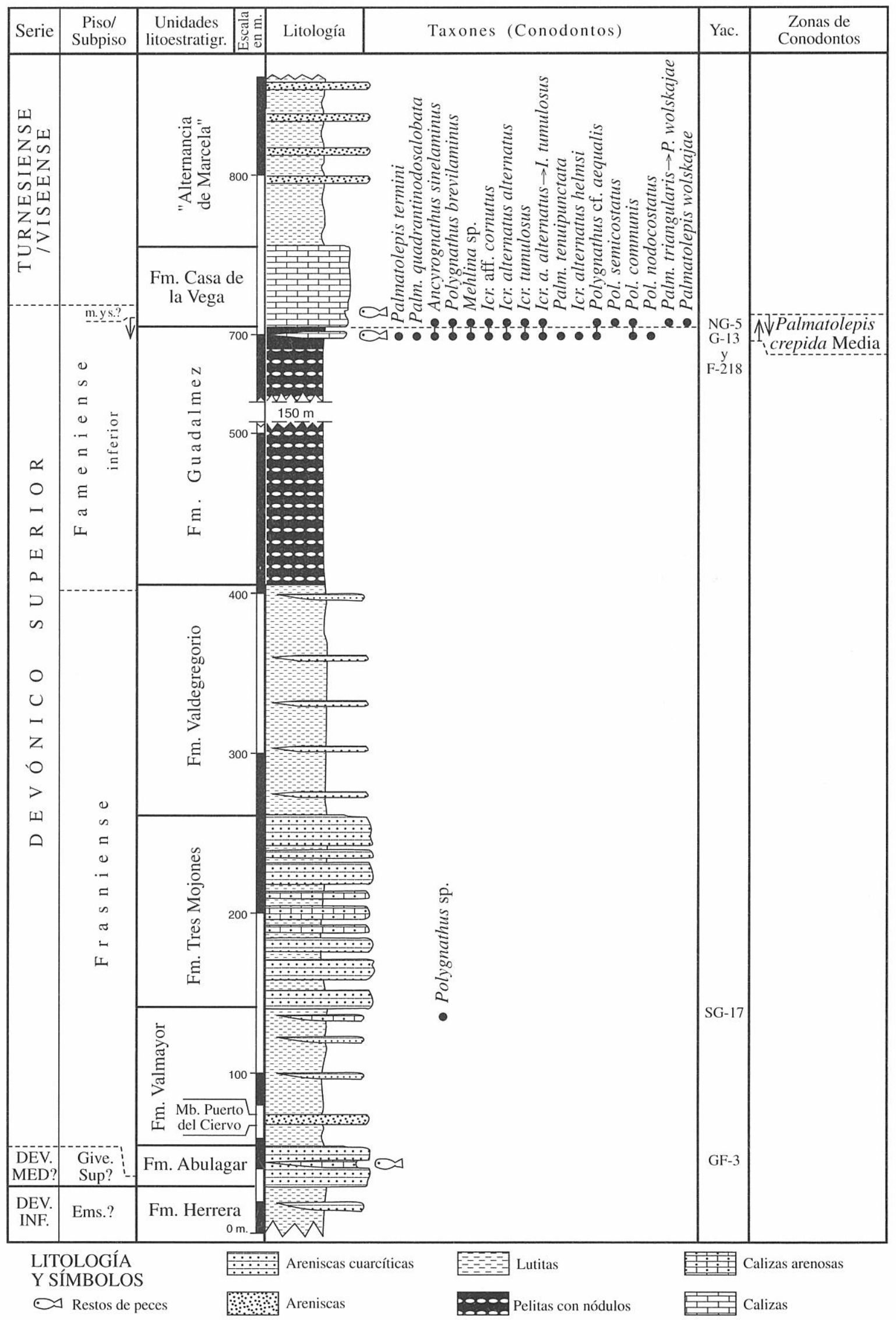

Figura 4. Columna sintética del Devónico Superior-Carbonífero del sinclinal de Guadalmez. Se indica la posición estratigráfica de las especies de conodontos citadas en el texto, salvo las del corte del Pantano de Mendoza, que se representan en la Fig. 3. 


\section{SINCLINAL DE GUADALMEZ}

Los niveles con conodontos proceden fundamentalmente del flanco norte del sinclinal de Guadalmez y uno, de su núcleo (Fig 1). Corresponden a muestras situadas en el tercio inferior de Formación Casa de la Vega, en el corte del Pantano de Mendoza (Fig. 3), y otros son muestras aisladas, integradas en una columna sintética, procedentes de la Formación Valmayor, del techo de la Formación Guadalmez en tránsito a la Formación Casa de la Vega y de los tramos basales de esta última unidad (Fig. 4). La cantidad de roca tratada ha sido de $1 \mathrm{~kg}$, resultando los conodontos escasos en la Formación Valmayor y más abundantes en el techo de la Formación Guadalmez y en la Formación Casa de la Vega, en especial en sus tramos basales.

En el techo de la Fm. Valmayor, el hallazgo de conodontos es irrelevante, un solo elemento asignado a Polygnathus sp. (Fig. 4).

La Fm. Guadalmez está formada por lutitas de color gris verdoso, azul y violeta, con intercalaciones centimétricas de areniscas de grano fino y niveles de nódulos fosilíferos. En su parte más alta se encuentran algunas capas centimétricas de calizas bioclásticas de las que se han obtenido conodontos, escamas, dientes y placas de peces. La muestra G-13, en el techo de la unidad (Fig. 4), contiene una predominancia de elementos I de Icriodus, en particular domina una forma transicional entre I. alternatus e I. tumulosus, siendo menos abundantes los ejemplares de Icriodus tumulosus Sánz López, que los de Icriodus alternatus alternatus Branson y Mehl (Lám. II, fig. 21); tambien hay elementos I de pequeña talla asignados a $I$. aff. cornutus Sannemann (Lám. II, fig. 18). El otro género dominante es Polygnathus los que, salvo un fragmento atribuído a Polygnathus aequalis Klapper y Lane, corresponden a Polygnathus brevilaminus Branson y Mehl (Lám. II, figs. 2-3). Destaca la escasez de elementos de Ancyrognathus y Palmatolepis, de los que se han encontrado A. sinelaminus (Branson y Mehl), elementos $\mathrm{Pa}$ de Palmatolepis quadrantinodosalobata Sannemann (Lám I, fig. 5) y Palmatolepis termini Sannemann (Lám. I, fig. 6), así como un elemento de Mehlina sp.

En un horizonte similar, situado al SO de Guadalmez, entre la trinchera del ferrocarril y una cantera al sur de río Guadalmez (Figs. 1 y 4, muestra F-218) los elementos de Palmatolepis corresponden a $P$. cf. wolskajae Ovnatanova y P. tenuipunctata Sannemann (Lám. I, fig. 5). También destaca la presencia de Icrioudus alternatus helmsi Sandberg y Dreesen (Lám. II, fig. 19), Polygnathus nodocostatus Branson y Mehl (Lám. II, fig. 6) y elementos de Mehlina sp. con patologías de tipo "descompositio" sensu Weddige (1990).

Palmatolepis termini es el taxón que define la base de la Zona de Palmatolepis crepida Media de Ziegler (1962), sin embargo, persiste hasta dentro de la Zona de $P$. crepida Superior de Ziegler y Sandberg (1990). La presencia de I. alternatus helmsi en la muestra F-218 restringiría el intervalo a la Zona de $P$. crepida Media del Fameniense inferior, según la distribución de dicha subespecie referida en Sandberg y Dreesen (1984).

La Fm. Casa de la Vega aflora exclusivamente en el sinclinal de Guadalmez y está constituída principalmente por calizas nodulosas, en la parte basal bioclásticas (calizas con cefalópodos) y en el resto micríticas; en determinados niveles son frecuentes las intercalaciones pelíticas. En el corte del Pantano de Mendoza (Fig. 3), la

\section{Lámina II}

1 Polygnathus cf. evidens Klapper y Lane, 1985. x 50, muestra V-428, Complejo vulcano-sedimentario de Chillón, sinclinal de Almadén.

2-3 Polygnathus brevilaminus Branson y Mehl, 1934. 2. x 80, muestra G-13; 3. x 60, muestra F-218, Fm. Casa de la Vega, sinclinal de Guadalmez.

4 Polygnathus semicostatus Branson y Mehl, 1934. x 40, muestra NG-5, Fm. Casa de la Vega, sinclinal de Guadalmez.

5 Polygnathus communis communis Branson y Mehl, 1934. x 110, muestra PG-6, Fm. Casa de la Vega, corte del Pantano de Mendoza, sinclinal de Guadalmez.

6 Polygnathus nodocostatus Branson y Mehl, 1934. x 30, muestra F-218, Fm. Casa de la Vega, sinclinal de Guadalmez.

7 Polygnathus procerus Sannemann, 1955. x 110, muestra PG-4, Fm. Casa de la Vega, sinclinal de Guadalmez.

8 Polygnathus lauriformis Dreesen y Dusar, 1974. x 40, muestra PG-5, Fm. Casa de la Vega, sinclinal de Guadalmez.

9 Polygnathus sp. x 50, muestra PG-4, Fm. Casa de la Vega, corte del Pantano de Mendoza, sinclinal de Guadalmez.

10 Bispathodus stabilis (Branson y Mehl, 1934). x 40, muestra PG-6, Fm. Casa de la Vega, corte del Pantano de Mendoza, sinclinal de Guadalmez.

11 Bispathodus bispathodus Ziegler, Sandberg y Austin, 1974. x 40, muestra PG-6, Fm. Casa de la Vega, corte del Pantano de Mendoza, sinclinal de Guadalmez.

12 Bispathodus costatus (Branson, 1934). x 50, muestra PG-6, Fm. Casa de la Vega, corte del Pantano de Mendoza, sinclinal de Guadalmez.

13-14 Bispathodus ultimus (Bischoff, 1957). 13. x 70; 14. x 80, muestra PG-6, Fm. Casa de la Vega, corte del Pantano de Mendoza, sinclinal de Guadalmez.

15-16 Icriodus sp. x 80, muestra NAL-7, Fm. Abulagar, sinclinal de Almadén.

17, 21 Icriodus alternatus alternatus Branson y Mehl, 1934. 17. x 90, muestra NG-5; 21. x 70, muestra G-13, Fm. Casa de la Vega, sinclinal de Guadalmez.

18 Icriodus aff. cornutus Sannemann, 1955. x 80, muestra F-218, Fm. Casa de la Vega, sinclinal de Guadalmez

19 Icriodus alternatus helmsi Sandberg y Dreesen, 1984. x 70, muestra F-218, Fm. Casa de la Vega, sinclinal de Guadalmez.

20 Icriodus tumulosus Sanz López, 1997. x 70, muestra PG-4, Fm. Casa de la Vega, sinclinal de Guadalmez. 
Lámina II

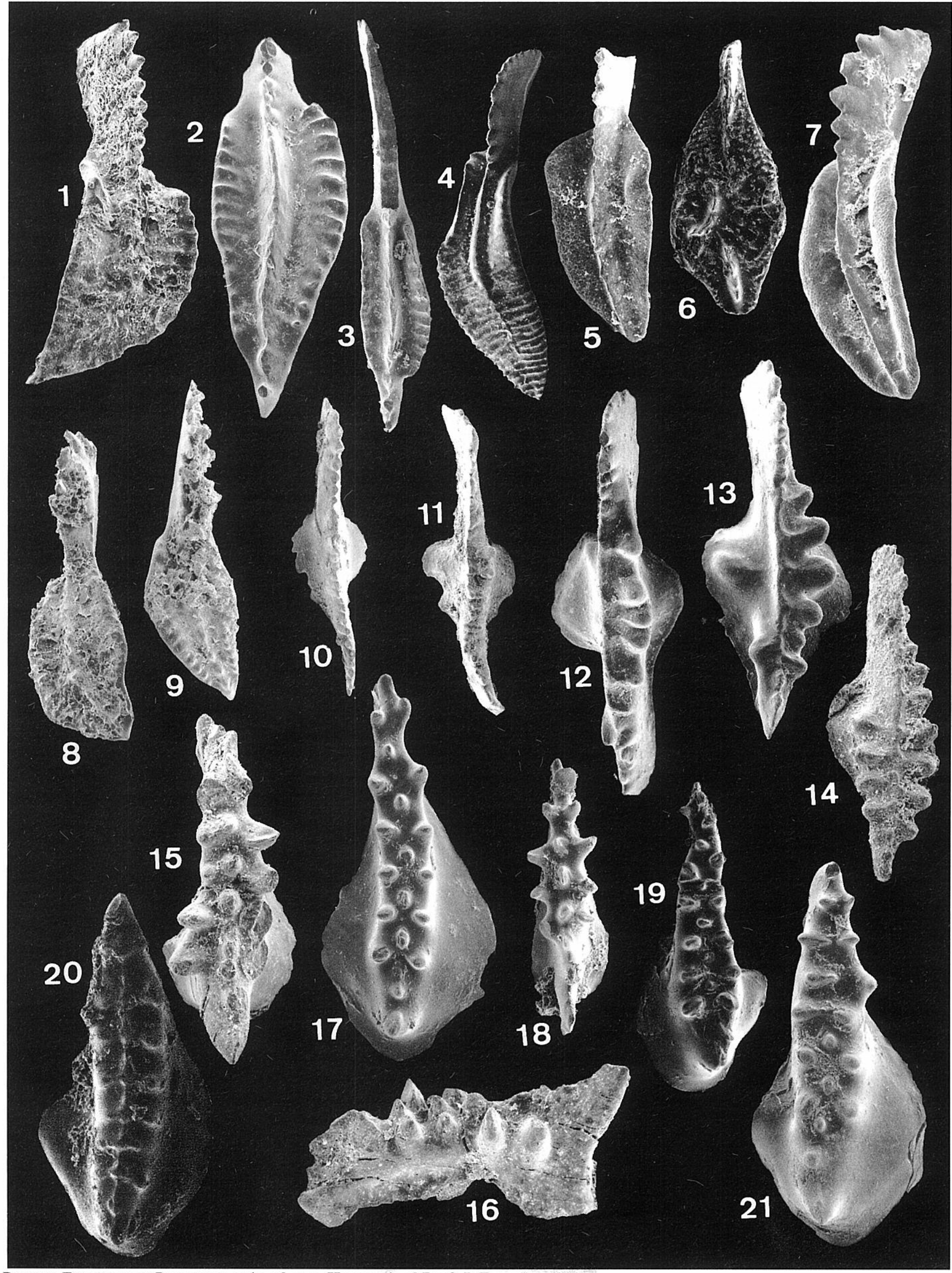


muestra PG-3, en la base de esta unidad, contiene Icriodus alternatus alternatus, I. tumulosus, formas transicionales entre I. alternatus e I. tumulosus y Polygnathus brevilaminus. Esta fauna es comparable, aunque menos diversa y abundante, a la obtenida en la localidad NG-5 (Fig. 4), también en los tramos basales de la Fm. Casa de la Vega, en donde dominan los elementos de Icriodus alternatus alternatus (Lám. II, fig. 17), ejemplares transicionales entre I. alternatus e $I$. tumulosus, I. tumulosus, escasos ejemplares de Icriodus alternatus helmsi (Lám. II, fig. 19) e I. aff. cornutus, una gran abundancia de Polygnathus brevilaminus, algunos ejemplares de $P$. communis Branson y Mehl, $P$. semicostatus Branson y Mehl (Lám. II, fig. 4) y un elemento de pequeña talla de Polygnathus aequalis. Otros taxones, tales como Mehlina sp. (Lám. I, figs 12-14), Ancyrognathus sinelaminus (Branson y Mehl) (Lám. I, fig. 15), Palmatolepis woslkajae (Lám. I, figs. 1-2) y una forma transicional entre Palmatolepis triangularis Sannemann y P. wolskajae (Lám. I, fig. 3), son escasos; esta fauna corresponde a un horizonte dentro de la Zona de $P$. crepida Media del Fameniense inferior (Ziegler y Sandberg, 1990) por la presencia de P. semicostatus junto a I. a. helmsi. Esta asignación permite precisar la correlación de la base de la Fm. Casa de la Vega, al igual que el techo de la Fm. Guadalmez, a la Zona de Palmatolepis crepida Media del Fameniense inferior.

El nivel PG-4 (Fig. 3) contiene Icriodus tumulosus (Lám. II, fig 20), Polygnathus sp. (Lám. II, fig. 9), $P$. procerus Sannemann (Lám. II, fig. 7), $P$. aequalis, formas transicionales entre Mehlina gradata (Youngquist) y Alternognathus pseudostrigosus (Dreesen y Dusar) y una forma juvenil de Ancyrognathus sinelaminus (Branson y Mehl). Esta muestra puede corresponder a un intervalo entre la Zona de Palmatolepis crepida Superior y la Zona de Palmatolepis crepida Más Superior de Ziegler y Sandberg (1990) por la presencia de Ancyrognathus sinelaminus. Polygnathus sp. es similar a formas encontradas en Pirineos por SanzLópez (1995) dentro de la Zona de P. rhomboidea Inferior de Sandberg y Ziegler (1973).

La muestra PG-5 procede de capas lenticulares de calizas bioclásticas y contiene Palmatolepis rhomboidea Sannemann (Lám. I, fig. 8), Palmatolepis minuta minuta Branson y Mehl (Lám. I, fig. 9), Polygnathus lauriformis Dreesen y Dusar (Lám. II, fig. 8), Mehlina cf. strigosa (Branson y Mehl) y otras formas entre Mehlina gradata y Alternognathus pseudostrigosus. La asociación es atribuída a la Zona de Palmatolepis rhomboidea de Sandberg y Ziegler (1973) del Fameniense inferior.

Una caliza nodular suprayacente, nivel PG-6, contiene Palmatolepis gracilis gracilis Branson y Mehl (Lám. I, fig. 10), Polygnathus communis communis Branson y Mehl (Lám. II , fig. 5), Bispathodus aculeatus aculeatus (Branson y Mehl), B. bispathodus Ziegler, Sandberg y Austin (Lám. II, fig. 11) B. costatus (Branson) (Lám. II, fig. 12), B. ultimus (Bischoff) (Lám. II, figs. 13-14), B. stabilis (Branson y Mehl) (Lám. II, fig. 10) Branmelha inornata (Branson y Mehl) (Lám. I, fig. 7), Mehlina gradata y $M$. strigosa . La muestra corresponde a un horizonte en el intervalo comprendido entre la Zona de Palmatolepis expansa Superior y la Zona de Siphonodella praesulcata Media del Fameniense superior (Ziegler y Sandberg, 1984).

Entre los niveles PG-5 y PG-6 es necesario algún fenómeno de condensación de la sedimentación o la existencia de una laguna estratigráfica ya que en unos 4 $m$ de sucesión deberían estar registradas las zonas de conodontos entre la de Palmatolepis marginífera hasta, al menos, la de Palmatolepis expansa Superior ( Ziegler y Sandberg, 1984) del Fameniense medio y casi todo el Fameniense superior.

Hacia el primer tercio de la Fm. Casa de la Vega, sobre la capa anterior y separada por unos dos metros de pizarras grises, se dispone una capa de calizas nodulosas de color marrón, de donde proceden las muestras PG-7 y G-3. La presencia de Gnathodus semiglaber Bischoff (Lám. I, fig. 16) y G. pseudosemiglaber Thompson y Fellows (Lám. I, figs. 17-18) en dichos niveles nos indica el Carbonífero Inferior. Gnathodus semiglaber se encuentra desde la Zona de Siphonodella isosticha-S. crenulata Superior (Sandberg et al., 1978) del Turnesiense superior hasta la Zona de Gnathodus austini o G. praebilineatus (Belka, 1985; Menéndez Álvarez, 1991), donde es raro, del Viseense medio. $G$. pseudosemiglaber se encuentra desde la Zona de Scaliognathus anchoralis-Doliognathus latus del Turnesiense terminal (Sandberg et al., 1978) hasta la Zona de Gnathodus austini o Zona de G. praebilineatus (Belka, 1985; Menéndez Álvarez, 1991) en el Viseense medio. Sin embargo, Perret (1993) indicó que la entrada de este último taxón en el Pirineo ocurre en la parte superior de su Zona de G. punctatus-Siphonodella, o sea un rango similar al señalado para $G$. semiglaber anteriormente. Lo más lógico, con una visión regional, es que ambas muestras pertenezcan al Turnesiense superior, en concreto a la Zona de $S$. anchoralis (Belka, 1985; Higgins, 1985), pero no hay criterios suficientes para afirmarlo de una forma tajante.

Entre los niveles PG-6 y PG-7, en unos $2 \mathrm{~m}$ de sucesión, deberían estar representadas las zonas de conodontos comprendidas entre la de Siphonodella praesulcata Superior (Ziegler y Sandberg, 1984) del Fameniense terminal y la de Siphonodella crenulata Inferior (Sandberg et al., 1978) del Turnesiense. Aunque no hay evidencias faunísticas suficientes, este tramo parece representar un nivel de condensación o falta de registro estratigráfico en torno al limíte DevónicoCarbonífero. Sin embargo, no se detectan mezclas de faunas en ninguna de las muestras estudiadas y cabe la posibilidad de que exista un hiato fosilizado, similar al citado en gran parte del dominio Astur-Leonés en la Zona Cantábrica (Menéndez Álvarez, 1991) o en Pirineos (Perret, 1993; Sanz-López, 1995).

\section{BIOFACIES DE CONODONTOS}

Las asociaciones de conodontos obtenidas para el Devónico pre-Fameniense son pobres en fauna y tienen 
muy poca diversidad. Los escasos elementos de Icriodus y Polygnathus proceden de niveles carbonatados ocasionales dentro de una sedimentación de plataforma siliciclástica y son indicativos de medios someros de alta a moderada energía.

Más significativas son las faunas de conodontos del Fameniense, con abundantes elementos y una diversidad relativamente baja. En las muestras del Fameniense inferior, en los tramos basales de la Fm. Casa de la Vega, hemos encontrado una predominancia de elementos de Icriodus y Polygnathus sobre los poco frecuentes elementos de Palmatolepis, Ancyrognathus y Mehlina. Corresponden a la biofacies de polignátidos-icriódidos de Sandberg y Dreesen (1984), que ha sido descrita alrededor de bioacumulaciones o en zonas retrabajadas por la acción de tempestades, extendiéndose a ambientes submareales someros, donde puede mezclarse con la biofacies de palmatolépidos-polignátidos de Sandberg y Dreesen (1984). En esta última biofacies se incluye la tafocenosis que hemos atribuído a la Zona de Palmatolepis rhomboidea, biofacies localizada en una posición más distante de la costa. La asociación del Fameniense superior parece denotar una mayor profundidad del medio, ya que se reconoce una biofacies de palmatolépidos-bispatódidos, considerada como de plataforma externa a cuenca (Sandberg y Ziegler, 1979). La evolución de las biofacies de conodontos durante el Fameniense indica un aumento en la profundidad y distancia a la costa en el medio de depósito, para el tercio inferior de la Fm. Casa de la Vega.

Mientras, las muestras del Carbonífero tan solo han proporcionado elementos de Gnathodus, género residente en una amplia variedad de habitats.

\section{PALEOTEMPERATURAS (CAI)}

Los conodontos, además de su utilidad biocronoestratigráfica y paleoecológica, son empleados en estudios de madurez termal. Ello es debido a que la materia orgánica presente en su composición cambia de color con el aumento de la temperatura. Estos cambios de color fueron cuantificados como índices numéricos o índices CAI (Epstein et al., 1977; Rejebian et al., 1987) y el índice de alteración del color de los elementos conodontales, junto a las alteraciones texturales que presentan (Sarmiento y García-López, 1996), son la respuesta al grado y tipo de metamorfismo presente en una zona. Cubren un intervalo térmico entre 50 y algo más de $600{ }^{\circ} \mathrm{C}$ y se han correlacionado con otros paleotermómetros orgánicos e inorgánicos.

En el área que nos ocupa (Fig. 1), Saupé et al. (1977) y Blachère (1978), a partir del índice de cristalinidad de la illita (IC) y de la reflectividad de la vitrinita (VR), indicaron condiciones diagenéticas en el flanco norte $\mathrm{y}$ núcleo del sinclinal de Almadén a anquizonales y epizonales en su flanco sur. Por su parte, Martínez Rius (1983) detectó la presencia de un débil clivaje en las pelitas del flanco sur del sinclinal de Guadalmez y Aller et al. (1986) observaron el desarrollo de clivaje en el flanco sur del sinclinal de Almadén y ocasionalmente en su flanco norte.

Sarmiento et al. (1999) mediante los datos de CAI obtenidos en calizas del Ordovícico superior, establecieron condiciones diagenéticas en el flanco norte del sinclinal de Almadén ( $\mathrm{CAI}=2,5 ; 3 ; 4$ y 6 ), con anomalías atribuídas a fluídos, probablemente relacionadas con el vulcanismo paleozoico prevarisco. Rocas de la misma edad en el sinclinal de Guadalmez, tanto en su flanco norte como en el sur, están tambien en el dominio de la diagénesis $(\mathrm{CAI}=3 ; 3,5)$.

De acuerdo con los datos del presente trabajo y teniendo en cuenta los datos anteriormente expuestos podemos indicar, para el sinclinal de Almadén, un metamorfismo creciente hacia el sur, localizándose la transición diagénesis-anquizona, en las rocas devónicas, entre el flanco norte y núcleo de esta estructura $(\mathrm{CAI}=4$; 4,5) y la epizona, en su flanco sur. La distribución del clivaje es consistente con la gradación del metamorfismo. Algunas anomalías en los valores del CAI sugieren la existencia de actividad hidrotermal. Para el sinclinal de Guadalmez se establecen condiciones diagenéticas $(\mathrm{CAI}=2 ; 2,5 ; 3)$, en rocas del Devónico y Carbonífero inferior, en el flanco norte y núcleo, con el desarrollo de un débil clivaje en el flanco sur asociado a valores del CAI comprendidos entre 3 y 3,5. Asimismo, la fase mineral de los conodontos (apatito) recristaliza con valores de $\mathrm{CAI}=4,5$.

\section{AGRADECIMIENTOS}

Nuestra gratitud a las Doctoras Graciela Sarmiento y Marie France Perret por las correcciones y sugerencias aportadas al manuscrito original. Este trabajo ha sido suvencionado por los Proyectos de la Dirección General de Enseñanza Superior del Ministerio de Educación DGEPB95-1047 y PB94-1324 y es una contribución al PICG n ${ }^{\circ}$ 421 "NorthGondwana Mid-Palaeozoic Bioevent/Biogeography patterns in relation to crustal dynamics".

\section{BIBLIOGRAFÍA}

Aller, J., Bastida, F., Ortega, E. y Pérez Estaún, A. 1986. Aportaciones al conocimiento estructural del Sinclinal de Almadén. Boletín del Instituto Geológico y Minero de España, 97, 608-621.

Belka, Z. 1985. Lower Carboniferous conodont biostratigraphy in the northeastern part of the MoraviaSilesia Basin. Acta Geologica Polonica, 35, 33-60.

Blachère, H. 1978. Etude géologique et minière de la région de Chillon-El Borracho (synclinal d' Almaden) Ciudad Real/Espagne. Tesis tercer ciclo. Universidad Paris-Sud D’Orsay, 154 pp. (inédita).

Bultynck, P. 1989. Conodonts from the La Grange Limestone (Emsian), Armorican Massif, NortWestern France. Courier Forschungs.-Institut Senckenberg, 117, 173-203.

Calvo, A. A. 1993. Conodontos del Devónico Inferior en el Sinclinal de Herrera del Duque (Badajoz SO. de España). 
Coloquios de Paleontología, 45, 9-228.

Epstein, A. G., Epstein, J. B. and Harris, L. D. 1977. Conodont color alteration-an index to organic metamophism. Geological Survey Profesional Paper, 995, 1-27.

García-Alcalde, J. L., Arbizu, M. A., Pardo Alonso, M. V. y García-López, S. 1984. El límite Devónico-Carbonífero en el área de Guadalmez-Santa Eufemia (Provs. de Ciudad Real y Córdoba, Sierra Morena, España). I Congreso Español de Geología. Segovia, 1, 421-430.

García-López, S., Sanz López, J. y Pardo Alonso, M. V. 1997. Conodontos de los sinclinales de Almadén y Guadalmez (Devónico-Carbonífero inferior), Zona Centroibérica meridional, España. XIII Jornadas de Paleontología, Libro de Resúmenes, 183-185.

García-López, S., Sanz López, J. y Pardo Alonso, M. V. 1998. Fammenian conodonts from the southern CentralIberian Zone (Iberian Variscan belt, SW Spain. In: ECOS VII Abstracts, Bologna-Modena (Ed. G. Bagnoli), 95-96.

Higgins, A. C. 1985. The Carboniferous system: Part 2Conodonts of the Silesian subsystem from Great Britain and Ireland. In: A stratigrapycal index of conodonts (Eds. A.C. Higgins and R.L. Austin). British Micropaleontological Society Series, 210-217.

Martínez Rius, A. 1983. Estudio geométrico de pliegues cónicos y su aplicación a la terminación occidental del sinclinal de Guadalmez, In: Libro Jubilar de J. M. RiosContribuciones sobre temas generales (Coord. J. A. Comba). 3, 177-192.

Menéndez Alvarez, J. R. 1991. Conodontos del Carbonífero Inferior y Medio de la Cordillera Cantábrica. Tesis Doctoral Universidad de Oviedo, 283 pp. (inédita).

Pardo Alonso, M. V. 1997. Geología del Devónico meridional de la Zona Centroibérica. Tesis Doctoral Universidad de Oviedo, 472 pp. (inédita).

Pardo Alonso, M. V. y García-Alcalde, J. L. 1984. Bioestratigrafía del Devónico de la región de Almadén (Ciudad Real). Trabajos de Geología, Universidad de Oviedo, 14, 79-120.

Pardo Alonso, M. V. y García-Alcalde, J. L. 1996. El Devónico de la Zona Centroibérica. Revista Española de Paleontología, $\mathbf{N}^{\mathbf{0}}$ Extraordinario, 72-81.

Perret, M. F. 1993. Recherches micropaleontologiques et biostratigraphiques (conodontes-foraminìferes) dans le Carbonifère Pyreneen. Strata, 2(21), 1-597.

Pushmann, H. 1970. Das Paläozoikum der Nördlichen "Sierra Morena" am Beispiel der Mulde von Herrera del Duque (Spanien). Geologie, 19, 309-329.

Rejebian, V. A., Harris, A. G. and Huebner, J. S. 1987. Conodont color and textural alteration: An index to regional metamorphism, and hydrotermal alteration. Geological Society of America Bulletin, 99, 471-479.

Sandberg, C. A. and Dreesen, R. 1984. Late Devonian icriodontid biofacies models and alternate shallow-water conodont zonation. Geological Society of America, Special Paper, 196, 143-179.

Sandberg, C. A. and Ziegler, W. 1973. Refinement of standard Upper Devonian conodont zonation based on sections in Nevada and West Germany. Geologica et Palaeontologica, 7, 97-122.

Sandberg, C. A. and Ziegler, W. 1979. Taxonomy and biofacies of important conodonts of Late Devonian styriacus-Zone, United States and Germany. Geologica et Palaeontologica, 13, 173-212.

Sandberg, C. A., Ziegler, W., Leuteritz, K. and Brill, S. M. 1978. Phyllogeny, speciation and zonation of Siphonodella (Conodonta, Upper Devonian and Lower Carboniferous). Newsletter Stratigraphy, 7, 102-120.

Sandberg, C. A., Ziegler, W., Dreesen, R. and Butler, J. L. 1988. Late Frasnian mass extinction: conodont event stratigraphy, global changes and possible causes. Courier Forschungs.-Institut Senckenberg, 102, 263-307.

Sanz López, J. 1995. Estratigrafía y Bioestratigrafía (Conodontos) del Silúrico superior-Carbonífero inferior del Pirineo central y oriental. Tesis Doctoral Universidad de Barcelona, 594 pp. (inédita).

Sarmiento, G. N. y García-López, S. 1996. El método del Indice de Alteración del Color (CAI) de los conodontos: limitaciones y posibilidades. Ejemplos de su aplicación en el Hercínico ibérico. Revista de la Sociedad Geológica de España, 9, 112-123.

Sarmiento, G. N., García-López, S. and Bastida, F. (1999). Conodont colour alteration index (CAI) of Upper Ordovician limestones from the Iberian Peninsula. Geologie en Minjbouw, 77, 77-92.

Saupé, F., Dunoyer de Segonzac, G. et Teichmüller, M. 1977. Etude du métamorphisme régional par la cristallinité de l'illite et la réflectance de la matière organique dans la zone du gisement de mercure d'Almaden (Province de Ciudad Real, Espagne). Sciences de la Terre, 21, 251-269.

Vergés, J. 1983. Estudio del complejo volcano-sedimentario del Devónico y de la estructura de la terminación oriental del sinclinal de Almadén (Ciudad Real). In: Libro Jubilar de J. M. Rios-Contribuciones sobre temas generales (Coord. J. A. Comba). 3, 215-229.

Verneuil, E. de et Barrande, J. 1855. Description des fossiles trouvés dans les terrains silurien et dévonien d'Almaden, d'una partie de la Sierra Morena et des Montagnes de Tolède. Bulletin Societe Géologique de France, 12, 9641025.

Weddige, K. 1990. Pathological conodonts. Courier Forschungs.-Institut Senckenberg, 118, 563-589.

Ziegler, W. 1962. Taxonomie und Phylogeny Oberdevonischer Conodonten und ihre stratigraphische Bedeutung. Abhandlungen des Hessischen Landesamtes für Bodenforshung, 38, 1-166.

Ziegler, W. and Sandberg, C. A. 1984. Palmatolepis-based revision of upper part of standard Late Devonian conodont zonation. Geological Society of America, Special Paper, 196, 179-194.

Ziegler, W. and Sandberg, C. A. 1990. The Late Devonian standard conodont zonation. Courier Forschungs.Institut Senckenberg, 121, 1-115. 